\title{
FITOSSOCIOLOGIA DO COMPONENTE ARBÓREO NUM REMANESCENTE DE FLORESTA OMBRÓFILA DENSA SUBMONTANA LIMÍTROFE À RESERVA BIOLÓGICA DO TINGUÁ, RIO DE JANEIRO
}

\author{
Felipe de Araújo Pinto Sobrinho ${ }^{1}$, Alexandre Gabriel Christo ${ }^{2}$, Rejan Rodrigues Guedes-Bruni ${ }^{3}$ \\ ${ }^{1}$ Eng. Florestal, M.Sc., Doutorando em Geografia Física, USP - felipesobrinho@usp.br \\ ${ }^{2}$ Eng. Agrônomo, M.Sc., ENBT - achristo@jbrj.gov.br \\ ${ }^{3}$ Bióloga, Dr ${ }^{\mathrm{a}}$., Instituto de Pesquisas Jardim Botânico do Rio de Janeiro - rbruni@ jbrj.gov.br \\ Recebido para publicação: 27/11/2007 - Aceito para publicação: 25/05/2008
}

\begin{abstract}
Resumo
O objetivo deste trabalho foi descrever os aspectos florísticos e estruturais do componente arbóreo em um fragmento alterado de Floresta Ombrófila Densa Submontana pertencente à Reserva Particular do Patrimônio Natural (RPPN) Centro de Ecologia e Cidadania do Tinguá (CEC Tinguá), no entorno da Reserva Biológica do Tinguá (RJ). Empregou-se para amostragem o método de parcelas, sendo incluídos todos os indivíduos com $5 \mathrm{~cm}$ ou mais de diâmetro a $1,3 \mathrm{~m}$ de altura do fuste (DAP). Foram amostrados 277 indivíduos, distribuídos em 21 famílias e 53 espécies, resultando em uma densidade total de 1.385 ind.ha $^{-1}$. As famílias com maior riqueza de espécies foram: Fabaceae, Lauraceae, Myrtaceae, Apocynaceae e Melastomataceae. A curva do coletor indicou saturação na amostragem. As espécies mais importantes foram: Pseudopiptadenia contorta, Lacistema pubecens, Apuleia leiocarpa e Aiouea saligna. O baixo índice de diversidade de Shannon, juntamente com as características estruturais e os grupos funcionais predominantes indicam que o remanescente encontra-se em estádio secundário de sucessão.

Palavras-chave: Floresta Ombrófila Densa Submontana; florística e estrutura; categorias sucessionais.
\end{abstract}

\begin{abstract}
Phytosociologic of a arboreus component of the remanent of Tropical Submontane Rainforest on the bordering of Tingua Biological Reserve, Rio de Janeiro. The objective of this work was to describe the floristic and structural aspects of the arboreal component in an altered Tropical Submontane Rainforest at Center of Ecology and Citizenship of the Tinguá (CEC Tinguá) Private Natural Heritage Reserves next to the Biological Reserve of Tinguá, Rio de Janeiro State. Using the plot method we included all individuals with diameter at $1,3 \mathrm{~m}$ (D.B.H.) $\geq 5 \mathrm{~cm}$. A total of 277 individuals were sampled, distributed in 21 families and 53 species, resulting in a total density of 1.385 ind.ha $^{-1}$. The species accumulation curve indicated saturation in the sampling. Fabaceae, Lauraceae, Myrtaceae Apocynaceae e Melastomataceae showed the highest values of species richness. The most important species were Pseudopiptadenia contorta, Lacistema pubecens, Apuleia leiocarpa and Aiouea saligna. The Low Shannon diversity index $\left(\mathrm{H}^{\prime}\right)$ together with the structural characteristics and the dominating functional groups of species indicating that the remainder finds itself in a secondary stage of succession.

Keywords: Dense Ombrophilous Submontane Forest; Floristic and structure; successional categories.
\end{abstract}

\section{INTRODUÇÃO}

No estado do Rio de Janeiro, projeções históricas estimam que 97\% da área original do território eram recobertos pela Mata Atlântica, o que corresponderia a aproximadamente $44.000 \mathrm{~km}^{2}$ de florestas (FUNDAÇÃO S.O.S. MATA ATLÂNTICA, 1993).

Atualmente, com uma população superior a 13 milhões de habitantes, sua área encontra-se reduzida a cerca de $19 \%$ (FUNDAÇÃO S.O.S. MATA ATLÂNTICA, 2002). Assim como no restante da 
Mata Atlântica, a floresta foi a principal fonte de recursos durante mais de quatrocentos anos, com a retirada de madeira e uso do solo nos diversos ciclos econômicos.

As grandes baixadas, sopés e encostas do estado, antes ocupados por densa floresta costeira, a partir do século XVI começaram a ceder lugar ao cultivo da cana-de-açúcar, primeiramente pelos arredores da Baía de Guanabara, seguida da Baixada Fluminense e posteriormente pelas encostas da Serra do Mar (GOLFARI; MOOSMAYER, 1980).

O município de Nova Iguaçu, inserido na Baixada Fluminense, atualmente preserva cerca de $38 \%$ de sua cobertura florestal original (CORREDORES DE BIODIVERSIDADE DA MATA ATLÂNTICA, 2006), e seus remanescentes totalizam área de 19.973 ha, que se encontram circunscritos à Floresta Ombrófila Densa (IBGE, 2004) e integram o Corredor de Biodiversidade da Serra do Mar.

Segundo Sobrinho (2007), 67\% do território do município foram transformados em área de preservação ambiental federal, estadual ou municipal. Destaca-se como a mais relevante, em função de sua reconhecida importância para a conservação da biodiversidade da porção do norte serrano da região de baixada, a Reserva Biológica do Tinguá, unidade federal subordinada ao IBAMA.

Os primeiros registros que documentaram a flora da serra do Tinguá foram realizados pelos naturalistas João Barbosa Rodrigues, Heinrich Karl Beirick, Frederik Christian Comes Raben, Auguste François Marie-Glaziou e Carl Friedrich Phillipp Von Martius, entre outros, durante o século XIX (CUSTÓDIO, 2007). Depois daquele período, houve muito poucos estudos sobre a flora arbustiva e arbórea na região (RODRIGUES, 1996; BRAZ et al., 2004; LIMA, 2002; SOBRINHO, 2007), a maioria atinente à área circunscrita à Reserva Biológica do Tinguá.

Até o momento não existem estudos fitossociológicos que caracterizem a flora arbustiva e arbórea dos fragmentos de floresta situados no entorno da ReBio, onde predomina a típica Floresta Ombrófila Densa Submontana (IBGE, 2004). Estudos recentes sobre florística e/ou fitossociologia (CARVALHO et al., 2007, em Rio Bonito; SOLÓRZANO et al., 2007; PEIXOTO et al., 2004, na cidade do Rio de Janeiro; Christo et al., 2009; CARVALHO et al., 2006; GUEDES-BRUNI et al., 2006; LIMA et al., 2006; PESSOA; OLIVEIRA, 2006, em Silva Jardim; dentre outros) buscaram caracterizar essas formações no estado do Rio de Janeiro.

Nesse contexto, este estudo teve como objetivo descrever a composição florística e a estrutura de um remanescente de Floresta Atlântica Submontana, que constitui uma Reserva Particular do Patrimônio Natural (RPPN), adjacente à Reserva Biológica do Tinguá, bem como contribuir para a formação de uma base de dados florístico-estruturais dessa região, ainda pouco estudada, mas que é de grande relevância, tanto para a diversidade biológica como para os recursos hídricos e bens culturais.

\section{MATERIAL E MÉTODOS}

\section{Caracterização da área de estudo}

A área inventariada situa-se na RPPN Centro de Ecologia e Cidadania Tinguá (22³5'S e $43^{\circ} 24^{\prime}$ W), em área limítrofe à circunscrição da Reserva Biológica do Tinguá (Figura 1), no bairro do Tinguá, extremo noroeste do município de Nova Iguaçu, Rio de Janeiro.

Nessa região ocorrem inúmeros fragmentos florestais, de vários tamanhos e diferentes estados de conservação. Muitos desses fragmentos, inclusive este em estudo, foram utilizados, num passado recente, para a retirada de madeiras destinadas à produção de carvão e indústria moveleira, extração de palmito e caça - processos que contribuíram para a redução populacional de muitas espécies da região.

A CEC Tinguá possui área de 16,5 ha, em altitude aproximada de $100 \mathrm{~m}$ s.n.m., sendo a única RPPN da região.

O clima regional é quente úmido, com estação seca pouco definida nos meses de julho e agosto, correspondendo ao tipo Am, segundo a classificação de Köppen. A temperatura média anual é de $21,6^{\circ} \mathrm{C}$ e a máxima absoluta é de $40,0{ }^{\circ} \mathrm{C}$, geralmente ocorrendo no mês de janeiro. A precipitação anual é de $2.099,3 \mathrm{~mm}$, sendo os meses mais chuvosos dezembro e janeiro (LIMA et al., 2002). A vegetação local caracteriza-se como Floresta Ombrófila Densa Submontana (IBGE, 2004).

\section{Amostragem}

Foram alocadas 10 parcelas de 10 × 20 ao longo da encosta do fragmento voltada para a fase Norte, totalizando uma área de amostragem de 0,2 ha. Como critério de inclusão, foram amostrados todos os indivíduos arbóreos vivos com diâmetro do caule a 1,30 m do solo (DAP) igual ou superiora $5 \mathrm{~cm}$. 


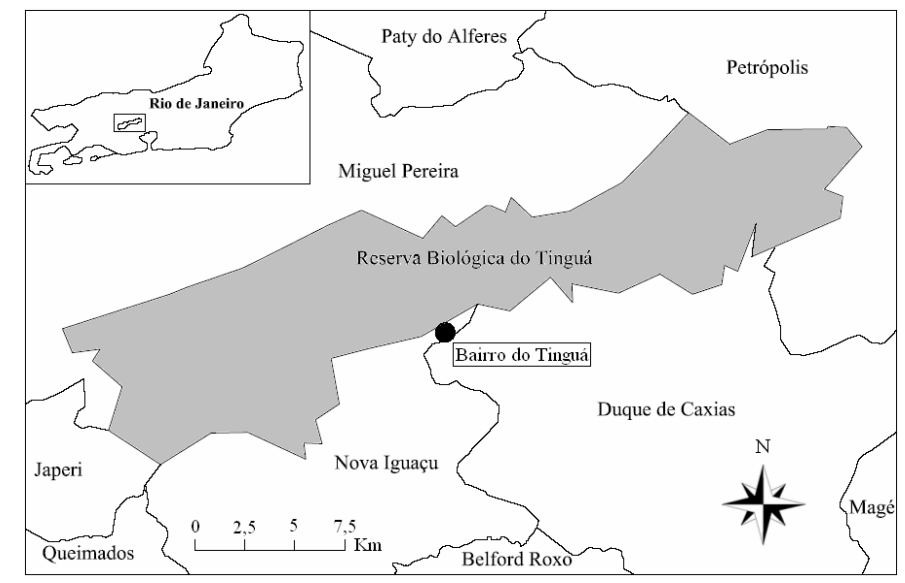

Figura 1. Mapa da Reserva Biológica do Tinguá, destacando o bairro do Tinguá, situado no extremo noroeste do município de Nova Iguaçu (RJ).

Figure 1. Map of Biological Reserve's Tingua, highlighting the District of Tingua located in the extreme northwest of the municipality of Nova Iguacu (RJ).

O material botânico coletado foi herborizado segundo métodos usuais em botânica, e a identificação realizada através de bibliografia especializada, bem como através de comparação com exsicatas do herbário Jardim Botânico do Rio de Janeiro (RB) e submetidas, quando necessário, a especialistas nos grupos taxonômicos complexos. O material testemunha foi depositado no Herbário VIC. O sistema de classificação adotado foi o APG II (2003). A nomenclatura taxonômica foi conferida através das bases de dados do MOBOT (www.tropicos.org) e do TreeAtlan ${ }^{1.0}$ (OLIVEIRA-FILHO, 2007). Através de pesquisa local com moradores, foi listado o nome popular das espécies amostradas.

\section{Análises}

Os parâmetros fitossociológicos estimados, segundo Mueller-Dombois; Ellenberg (1974), foram densidade, frequência e dominância, sobre os quais foi calculado o valor de importância. Para calcular os referidos parâmetros, utilizou-se o software Fitopac (SHEPHERD, 1996).

A diversidade da área foi estimada com base no índice de Shannon (H') (MAGURRAN, 1988), e a equidade (J), segundo Pielou (1975).

Através do ajuste de uma reta por regressão linear simples (ZAR, 1999), com base no índice de riqueza de táxons (WHITTAKER, 1975) acumulado em função do número de observações (LOSS; SILVA, 2005), foi calculada a curva de esforço amostral para a área, conforme utilizado por Christo et al., 2009.

As espécies foram classificadas em grupos ecológicos de acordo com Gandolfi et al. (1995), sendo distribuídas nas seguintes categorias sucessionais: Pioneiras (P), Secundárias iniciais ( $\mathrm{Si}$ ) e Secundárias tardias (St). Tomaram-se como base, trabalhos com enfoque semelhante, desenvolvidos nas regiões sob o Domínio Atlântico (CARVALHO et al., 2006; PEIXOTO et al., 2004; SANTANA, 2000; OLIVEIRA, 2002; COSTA; MANTOVANI, 1995). Algumas espécies, cuja classificação sucessional não foi encontrada em literatura ou cujos dados não coincidiam nas diferentes literaturas, tiveram sua classificação baseada em observações de campo.

\section{RESULTADOS E DISCUSSÃO}

Foram amostradas 53 espécies, subordinadas a 21 famílias (Tabela 1). O ajuste altamente significativo da reta em função da riqueza dos pontos de amostragem $(\mathrm{p}<0,0001)$ indicou que a riqueza florística naquele trecho de floresta, restrita à encosta norte do fragmento, foi bem representada (Figura 2). Apesar desse resultado, não se pode inferir que a amostragem foi representativa da diversidade de espécies de toda a 
comunidade, pois, segundo Schilling; Batista (2008), uma lista de composição não irá representar o sistema, pois as interações espaço-temporais tornam essa lista variável no espaço e no tempo.

Tabela 1. Listagem das espécies amostrados na RPPN CEC Tinguá, Nova Iguaçu (RJ), com as respectivas categorias sucessionais $(\mathrm{CS})$. Pi: pioneira; Si: secundária inicial; St: secundária tardia; S/c: sem classificação).

Table 1. Checklist of sampled species at RPPN CEC Tingua, Nova Iguaçu (RJ), with respective sucessional categories (CS). Pi: pioneer species; Si: early secondary species; St: late secondary species; S/c: without data).

\begin{tabular}{|c|c|c|}
\hline Espécie & Nome local & CS \\
\hline \multicolumn{3}{|l|}{ Anacardiaceae } \\
\hline Astronium graveolens Jacq. & aderne & $\mathrm{Si}$ \\
\hline Tapirira guianensis Aubl. & pau-pombo & $\mathrm{Si}$ \\
\hline \multicolumn{3}{|l|}{ Annonaceae } \\
\hline Guatteria candolleana Schltdl. & imbiu-preto/conde & $\mathrm{S} / \mathrm{c}$ \\
\hline Xylopia brasiliensis Spreng. & pindaíba & $\mathrm{Pi}$ \\
\hline \multicolumn{3}{|l|}{ Apocynaceae } \\
\hline Geissospermum vellosii Allemão & pau-pereira & $\mathrm{Si}$ \\
\hline Himatanthus lancifolius (Müll. Arg.) Woodson & aguniada & St \\
\hline Malouetia arborea (Vell.) Miers & esperta-roxa & $\mathrm{Si}$ \\
\hline Tabernaemontana laeta Mart. & esperta & $\mathrm{Si}$ \\
\hline \multicolumn{3}{|l|}{ Arecaceae } \\
\hline Astrocaryum aculeatissimum (Schott) Burret & irí & St \\
\hline \multicolumn{3}{|l|}{ Asteraceae } \\
\hline Vernonia discolor (Spreng.) Less. & cambará & $\mathrm{Pi}$ \\
\hline Stifftia chrysantha Mikan. & candeia/rabo-de-cutia & $\mathrm{S} / \mathrm{c}$ \\
\hline \multicolumn{3}{|l|}{ Bignoniaceae } \\
\hline Cybistax antisyphilitica (Mart.) Mart. & cinco-folhas/ipê & $\mathrm{Si}$ \\
\hline Jacaranda puberula Cham. & carobinha & $\mathrm{Si}$ \\
\hline Sparattosperma leucanthum (Vell.) Schum. & cinco-folhas/ipê-branco & $\mathrm{Si}$ \\
\hline \multicolumn{3}{|l|}{ Lacistemataceae } \\
\hline Lacistema pubescens Mart. & sabonete & $\mathrm{Si}$ \\
\hline \multicolumn{3}{|l|}{ Lauraceae } \\
\hline Aiouea saligna Meisn. & canela-parda & St \\
\hline Licaria armenica (Nees) Kosterm. & - & St \\
\hline Nectandra membranacea (Sw.) Griseb. & canela & St \\
\hline Ocotea lancifolia (Schott) Mez & canela & St \\
\hline Ocotea schottii (Meisn.) Mez & canela & St \\
\hline Ocotea $\mathrm{sp}$. & canela & $\mathrm{S} / \mathrm{c}$ \\
\hline \multicolumn{3}{|l|}{ Lecythidaceae } \\
\hline Lecythis pisonis Cambess. & sapucaia & St \\
\hline \multicolumn{3}{|l|}{ Fabaceae } \\
\hline Anadenanthera sp. & angico-rosa & $\mathrm{Si}$ \\
\hline Apuleia leiocarpa (Vogel) J.F. Macbr. & garapa/pau-preguiça & $\mathrm{Si}$ \\
\hline Machaerium brasiliense Vogel & - & $\mathrm{Si}$ \\
\hline Myrocarpus frondosus Allemão & óleo-pardo & St \\
\hline Piptadenia gonoacantha (Mart.) J.F. Macbr. & pau-jacaré/jacaré & $\mathrm{Pi}$ \\
\hline $\begin{array}{l}\text { Pseudopiptadenia contorta (DC.) G.P. } \\
\text { Lewis \& M.P. Lima }\end{array}$ & angico-cambuí & $\mathrm{Si}$ \\
\hline
\end{tabular}




\begin{tabular}{|c|c|c|}
\hline \multicolumn{3}{|l|}{ Melastomataceae } \\
\hline Miconia albicans (Sw.) Triana & - & $\mathrm{S} / \mathrm{c}$ \\
\hline Miconia cinnamomifolia (DC.) Naudin & jacatirão & $\mathrm{Pi}$ \\
\hline Miconia lepidota Schrank \& Mart. ex DC. & - & $\mathrm{S} / \mathrm{c}$ \\
\hline Miconia prasina (Sw.) DC. & - & $\mathrm{Si}$ \\
\hline \multicolumn{3}{|l|}{ Meliaceae } \\
\hline Cabralea canjerana (Vell.) Mart. & canjerana & $\mathrm{St}$ \\
\hline \multicolumn{3}{|l|}{ Moraceae } \\
\hline Brossimum guianensis (Aubl.) Huber & & $\mathrm{Si}$ \\
\hline \multicolumn{3}{|l|}{ Myrcinaceae } \\
\hline Myrcine ferruginea (Ruiz \& Pav.) Mez & copororoca & $\mathrm{Si}$ \\
\hline \multicolumn{3}{|l|}{ Myrtaceae } \\
\hline Eugenia tinguyensis Cambess. & - & $\mathrm{St}$ \\
\hline Eugenia villae-novae Kiaersk. & - & $\mathrm{St}$ \\
\hline Myrcia splendens (Sw.) DC. & - & $\mathrm{Si}$ \\
\hline Myrciaria floribunda (West ex Wild) Berg & - & $\mathrm{Si}$ \\
\hline Psidium guineense $\mathrm{Sw}$. & araçá & $\mathrm{St}$ \\
\hline \multicolumn{3}{|l|}{ Nyctaginaceae } \\
\hline Guapira opposita (Vell.) Reitz & cebola/cebolinha & $\mathrm{Si}$ \\
\hline \multicolumn{3}{|l|}{ Proteaceae } \\
\hline Roupala sculpta Sleumer & carne-de-vaca & $\mathrm{St}$ \\
\hline \multicolumn{3}{|l|}{ Rubiaceae } \\
\hline Psycotria velloziana Benth. & - & $\mathrm{St}$ \\
\hline Rubiaceae 1 & - & St \\
\hline \multicolumn{3}{|l|}{ Rutaceae } \\
\hline Citrus aurantiifolia (Christm.) & galego & $\mathrm{S} / \mathrm{c}$ \\
\hline Zanthoxylum rhoifolium Lam. & mamica-de-porca & $\mathrm{Pi}$ \\
\hline \multicolumn{3}{|l|}{ Sapindaceae } \\
\hline Allophylus sp. & - & $\mathrm{Si}$ \\
\hline Cupania oblongifolia Mart. & camboatá & $\mathrm{Si}$ \\
\hline Cupania racemosa (Vell.) Radlk. & camboatazinho & $\mathrm{St}$ \\
\hline \multicolumn{3}{|l|}{ Siparunaceae } \\
\hline Siparuna guianensis Aubl. & negramina & $\mathrm{Si}$ \\
\hline \multicolumn{3}{|l|}{ Indeterminada } \\
\hline Indet sp1 & - & $\mathrm{S} / \mathrm{c}$ \\
\hline Indet sp2 & - & $\mathrm{S} / \mathrm{c}$ \\
\hline Indet sp3 & piquiá & $\mathrm{Si}$ \\
\hline
\end{tabular}

As famílias que apresentaram maior número de indivíduos foram Fabaceae, com 48 indivíduos amostrados, Lacistemataceae (44), Lauraceae (40) e Melastomataceae (16), que, juntas, somam 53,4\% do total de indivíduos amostrados para a área. Dentre as espécies que mais contribuíram para a incidência dessas famílias, destacam-se, em Fabaceae, Pseudopiptadenia contorta e Apuleia leiocarpa, ambas classificadas como secundárias iniciais; Lacistemataceae foi representada apenas pela espécie secundária inicial Lacistema pubecens; em Lauraceae destacou-se Aiouea saligna, classificada como secundária tardia; e em Melastomataceae destacou-se Miconia lepidota, cuja classificação sucessional não foi determinada.

As famílias com maior riqueza específica (Tabela 1) foram Lauraceae e Fabaceae, com seis espécies cada, Myrtaceae (cinco), Apocynaceae e Melastomataceae (quatro). Essas famílias também se destacaram como as cinco mais ricas no trabalho de Rodrigues (1996), estudando a Vegetação Ombrófila Densa Submontana do interior da Reserva Biológica do Tinguá. Guedes (1988), estudando um trecho de floresta perturbada em Magé (RJ), encontrou essas cinco famílias entre as seis mais ricas em número de espécies. 


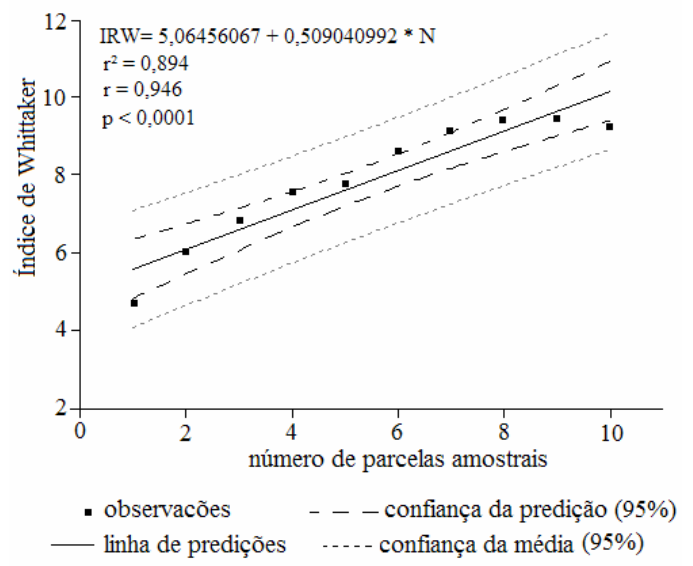

Figura 2. Curva do coletor para as espécies amostradas na RPPN (CEC Tinguá), Bairro do Tinguá, Nova Iguaçu (RJ).

Figure 2. Curve of the collector for the species sampled in RPPN (CEC Tingua), District of Tingua, Nova Iguacu (RJ).

Myrtaceae, Fabaceae, Lauraceae e Euphorbiaceae têm sido constantemente citadas entre as famílias com maior riqueza de espécies em estudos florísticos e/ou fitossociológicos realizados em diferentes regiões no estado do Rio de Janeiro (PROGRAMA MATA ATLÂNTICA, 1992; OLIVEIRA et al., 1995; GUEDES-BRUNI et al., 1997; PESSOA et al., 1997; KURTZ; ARAÚJO, 2000; PEIXOTO et al., 2004; CARVALHO et al., 2006).

Comparando a listagem florística desse estudo com o realizado por Rodrigues et al. (1996), em uma área de floresta submontana no interior da ReBio do Tinguá, em local com características geográficas bem próximas às do presente estudo, de um total de 189 espécies encontradas pelo autor, apenas 12 foram comuns ao presente trabalho: Astronium graveolens, Cabralea canjerana, Pseudopiptadenia contorta, Cupania oblongifolia, Guapira opposita, Lacistema pubecens, Miconia prasina, Tabernaemontana laeta, Astrocaryum aculeatissimum, Mirocarpus frondosus, Nectandra membranacea e Ocotea schottii, sendo a maioria dessas espécies características de etapas iniciais de sucessão florestal. Essa diferença florística se justifica, em parte, devido ao maior grau de conservação da área situada no interior da ReBio.

Analisando as espécies arbóreas encontradas em fragmentos florestais no entorno da ReBio do Tinguá por Sobrinho et al. (2007), ao estudar o uso de recursos vegetais por moradores locais, muitas espécies citadas para diversos usos foram amostradas no presente inventário. Para uso medicinal: Cybistax antisyphilitica (ipê-cinco-folhas), Geissospermum vellosei (pau-pereira), Jacaranda puberula (carobinha); alimentício: Lecitis pisonis (sapucaia), Astrocarium aculeatissimum (irí); construção: Apuleia leiocarpa (garapa), Astronium graveolens (aderne), Cabralea canjerana (canjerana), Xilopia sericea (imbiu), Myrocarpus frondosus (óleo-pardo), Stifftia grazielae (candeia-rabo-de-cutia), Pseudopiptadenia contorta (angico-cambuí), Miconia cinnamomifolia (jacatirão), Malouetia arborea (esperta-roxa) e Cupania racemosa (camboatá), entre outras. Esses dados indicam a importância da conservação dos remanescentes locais, uma vez que eles, apesar do histórico de perturbação sofrida em passado recente, ainda detêm grande importância ecológica, econômica, social e cultural.

$\mathrm{O}$ valor do índice de diversidade encontrado no presente estudo foi 3,423. Para as áreas antropizadas descritas na tabela 2 , o índice variou de 1,69 a 3,66. Índices maiores que 4,05 foram encontrados para florestas em melhor estado de conservação (CHRISTO et al., 2009; GUEDES-BRUNI et al., 2006; KURTZ; ARAÚJO, 2000; GUEDES-BRUNI et al., 1997; RODRIGUES, 1996).

Kurtz; Araújo (2000), analisando o índice de diversidade de Shannon (H') em 14 estudos realizados na Mata Atlântica do estado do Rio de Janeiro e São Paulo, encontraram uma variação no índice de 1,69 a 4,40, sendo que os baixos índices foram associados a características edáficas e de perturbação antrópica. 
Tabela 2. Tabela comparativa de alguns estudos realizados no estado do Rio de Janeiro. DI: diâmetro de inclusão das espécies; N: número de espécies amostradas; H’: Índice de diversidade de Shannon.

Table 2. Compactive table of some studies performed in the state of Rio de Janeiro. DI: diameter of inclusion of the species; $\mathrm{N}$ : number of species sampled; H': Shannon diversity index.

\begin{tabular}{lccccc}
\hline Local & Autor & Método & DI $(\mathbf{c m})$ & $\mathbf{N}$ & H' \\
\hline Bairro do Tinguá* & Presente estudo & Parcelas & 5 & 53 & 3,42 \\
Silva Jardim & Guedes-Bruni et al., 2006 & Parcelas & 5 & 174 & 4,20 \\
Silva Jardim & Christo et al., 2009 & Parcelas & 5 & 129 & 4,22 \\
Serra da Capoeira Grande* & Peixoto et al., 2005 & Quadrantes & 5 & 44 & 2,42 \\
Cachoeira de Macacu & Kurtz \& Araújo, 2000 & Quadrantes & 5 & 174 & 4,57 \\
Macaé de Cima & Guedes-Bruni et al., 1997 & Parcelas & 5 & 189 & 4,05 \\
Macaé de Cima* & Pessoa et al., 1997 & Parcelas & 5 & 157 & 3,66 \\
Nova Iguaçu & Rodrigues, 1996 & Parcelas & 2,55 & 189 & 4,36 \\
Magé* & Guedes, 1988 & Parcelas & 5 & 100 & 1,69 \\
\hline
\end{tabular}

*Áreas alteradas antropicamente.

A equidade (J) apresentou valor de 0,862 , indicando que o remanescente estudado detém elevada heterogeneidade florística.

Para o cálculo da estrutura arbórea, foram mensurados 277 indivíduos vivos, perfazendo uma densidade total (DT) de 1.385 indivíduos por hectare, diâmetro médio de 11,91 cm e área basal por hectare de $22.136 \mathrm{~m}^{2}$. A altura média da população foi de 8,51 metros, com presença de indivíduos arbóreos emergentes alcançando mais de 25 metros de altura.

A distribuição diamétrica (Figura 3) apresentou o padrão "J" invertido (HARPER, 1990), com a maioria dos indivíduos amostrados $(56,3 \%)$ inserida na primeira classe de diâmetro $(5-10 \mathrm{~cm})$, o que indica acentuado incremento de indivíduos jovens na comunidade. As espécies Apuleia leiocarpa e Myrocarpus frondosus apresentaram todos os indivíduos representados por diâmetros menores que $30 \mathrm{~cm}$, fato esse que pode ser explicado em função da importância madeireira dessas espécies, o que resultou em uma exploração excessiva delas em um passado recente.

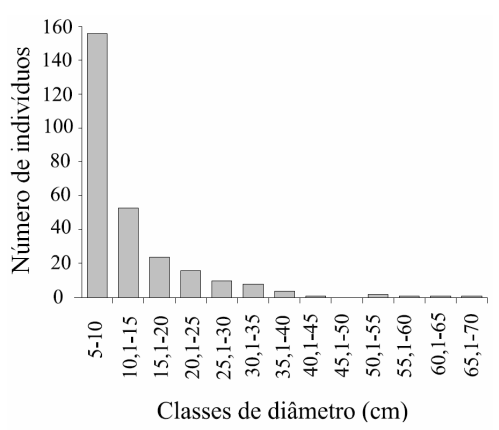

Figura 3. Distribuição de frequência das classes de diâmetro dos indivíduos amostrados na RPPN (CEC Tinguá), bairro do Tinguá, Nova Iguaçu (RJ).

Figure 3. Diametric classes distribution frequency of the individuals sampled in RPPN (CEC Tingua), district of Tingua, Nova Iguaçu (RJ).

Quanto à densidade relativa (DR), destacaram-se Lacistema pubescens (15,88\%), Pseudopiptadenia contorta (7,22\%), Aiouea saligna (6,14\%), Apuleia leiocarpa (5,78\%) e Siparuna guianensis $(5,05 \%)$. Com exceção de Aiouea saligna, classificada como secundária tardia, todas as demais são secundárias iniciais. Das 53 espécies encontradas na amostra (incluindo as morfoespécies), 13 possuem o menor valor $(0,36 \%)$ encontrado para o parâmetro densidade relativa, todas contribuindo com apenas um indivíduo na amostragem.

Lacistema pubescens, típica secundária inicial, caracterizou-se pela presença de indivíduos jovens, todos com diâmetros inferiores a $15 \mathrm{~cm}$ e bem distribuídos por toda a área amostral. GuedesBruni (1998) destaca essa espécie como de ampla distribuição geográfica ao longo da faixa oriental brasileira, sendo espécie característica de ocupação e propagação em áreas alteradas. 
Nenhuma dessas cinco espécies de maior densidade foi encontrada por Rodrigues (1996) apesar da proximidade e semelhança geográfica entre as duas áreas estudadas -, que relaciona entre as mais abundantes: Guapira opposita, Piper rivinoides, Geonoma fiscellaria, Euterpe edulis e Psychotria glaziovii.

As espécies que se destacaram quanto ao VI (Tabela 3) foram Pseudopiptadenia contorta e Lacistema pubecens, sendo que Pseudopiptadenia contorta teve seu alto valor de VI (31.67\%) em função da grande dominância de seus indivíduos. Por outro lado, Lacistema pubecens $(\mathrm{VI}=26.58 \%)$ se destacou devido à elevada densidade dos indivíduos.

Tabela 3. Relação das espécies amostradas na RPPN (CEC Tinguá), bairro do Tinguá, Nova Iguaçu (RJ), em ordem decrescente de valor de importância (VI). N.I: número de indivíduos; DR: densidade relativa; FR: frequência relativa; DoR: dominância relativa.

Table 3. Relation of species sampled in RPPN (CEC Tinguá), district of Tingua, Nova Iguaçu (RJ), in descending order of value of impotance (VI). N.I: number of species; DR: relative density; FR: relative frequency; DoR: relative dominance.

\begin{tabular}{|c|c|c|c|c|c|}
\hline Espécie & N.I & DR & FR & DoR & VI \\
\hline Pseudopiptadenia contorta & 20 & 7.22 & 4.61 & 19.85 & 31.67 \\
\hline Lacistema pubecens & 44 & 15.88 & 6.58 & 4.12 & 26.58 \\
\hline Apuleia leiocarpa & 16 & 5.78 & 5.26 & 8.16 & 19.20 \\
\hline Aiouea saligna & 17 & 6.14 & 5.92 & 5.40 & 17.46 \\
\hline Miconia lepidota & 8 & 2.89 & 3.95 & 8.33 & 15.17 \\
\hline Siparuna guianensis & 14 & 5.05 & 4.61 & 1.93 & 11.59 \\
\hline Vernonia diffusa & 8 & 2.89 & 3.95 & 4.23 & 11.06 \\
\hline Sparatosperma leucantum & 7 & 2.53 & 2.63 & 5.56 & 10.72 \\
\hline Astrocarium aculeatissimum & 9 & 3.25 & 3.29 & 3.42 & 9.96 \\
\hline Astronium graveolens & 11 & 3.97 & 4.61 & 1.30 & 9.87 \\
\hline Miconia cinnamomifolia & 4 & 1.44 & 2.63 & 5.54 & 9.62 \\
\hline Ocotea sp. & 7 & 2.53 & 3.29 & 2.03 & 7.85 \\
\hline Cabralea canjerana & 9 & 3.25 & 1.97 & 1.89 & 7.11 \\
\hline Tabernaemontana laeta & 6 & 2.17 & 1.97 & 2.92 & 7.06 \\
\hline Machaerium brasiliense & 5 & 1.81 & 2.63 & 2.59 & 7.03 \\
\hline Eugenia tinguiensis & 9 & 3.25 & 1.97 & 1.41 & 6.64 \\
\hline Licaria armenica & 8 & 2.89 & 2.63 & 0.79 & 6.31 \\
\hline Xilopia sericea & 4 & 1.44 & 1.97 & 1.40 & 4.82 \\
\hline Psycotria velloziana & 4 & 1.44 & 2.63 & 0.35 & 4.42 \\
\hline Jacaranda puberula & 4 & 1.44 & 2.63 & 0.30 & 4.38 \\
\hline Myrocarpus frondosus & 5 & 1.81 & 1.32 & 0.88 & 4.00 \\
\hline Cupania racemosa & 1 & 0.36 & 0.66 & 2.91 & 3.93 \\
\hline Lecitis pisonis & 4 & 1.44 & 1.32 & 1.11 & 3.87 \\
\hline Cupania oblongifolia & 2 & 0.72 & 1.32 & 1.80 & 3.84 \\
\hline Guatteria candolleana & 3 & 1.08 & 1.97 & 0.63 & 3.69 \\
\hline Alophyllus sp. & 3 & 1.08 & 1.32 & 0.98 & 3.38 \\
\hline Nectandra membranacea & 3 & 1.08 & 1.32 & 0.75 & 3.15 \\
\hline Miconia prasina & 3 & 1.08 & 1.32 & 0.40 & 2.79 \\
\hline Guapira opposita & 3 & 1.08 & 1.32 & 0.24 & 2.64 \\
\hline Psidium guineesis & 2 & 0.72 & 1.32 & 0.39 & 2.43 \\
\hline Rubiaceae sp. & 4 & 1.44 & 0.66 & 0.28 & 2.38 \\
\hline Ocotea schottii & 2 & 0.72 & 1.32 & 0.32 & 2.36 \\
\hline Piptadenia gonoacantha & 1 & 0.36 & 0.66 & 1.28 & 2.30 \\
\hline Eugenia villae-novae & 1 & 0.36 & 0.66 & 1.22 & 2.24 \\
\hline Indet sp.1 & 2 & 0.72 & 0.66 & 0.85 & 2.23 \\
\hline Himatanthus lancifolius & 2 & 0.72 & 1.32 & 0.17 & 2.21 \\
\hline Indet sp. & 2 & 0.72 & 1.32 & 0.15 & 2.19 \\
\hline Brossimum guianensis & 2 & 0.72 & 0.66 & 0.73 & 2.11 \\
\hline Ocotea lancifolia & 3 & 1.08 & 0.66 & 0.33 & 2.07 \\
\hline
\end{tabular}




\begin{tabular}{llllll}
\hline Geissospermum vellosei & 2 & 0.72 & 0.66 & 0.65 & 2.02 \\
Malouetia arborea & 1 & 0.36 & 0.66 & 0.63 & 1.65 \\
Anadenanthera sp. & 1 & 0.36 & 0.66 & 0.52 & 1.54 \\
Stifftia chrysantha Mikan. & 1 & 0.36 & 0.66 & 0.22 & 1.24 \\
Myrcine ferruginea & 1 & 0.36 & 0.66 & 0.22 & 1.24 \\
Indet sp. & 1 & 0.36 & 0.66 & 0.15 & 1.16 \\
Zanthoxilum rhoifolium & 1 & 0.36 & 0.66 & 0.12 & 1.14 \\
Cybistax antisiphilitica & 1 & 0.36 & 0.66 & 0.10 & 1.11 \\
Citrus aurantiifolia & 1 & 0.36 & 0.66 & 0.10 & 1.11 \\
Miconia albicans & 1 & 0.36 & 0.66 & 0.09 & 1.11 \\
Tapirira guianensis & 1 & 0.36 & 0.66 & 0.08 & 1.10 \\
Myrcia splenddens & 1 & 0.36 & 0.66 & 0.08 & 1.09 \\
Roupala sculpta & 1 & 0.36 & 0.66 & 0.07 & 1.08 \\
Myrciaria floribunda & 1 & 0.36 & 0.66 & 0.05 & 1.06 \\
\hline
\end{tabular}

Rodrigues (1996) destaca como as mais importantes de sua área de estudo Guapira opposita, Piper rivinoides, Geonoma fiscellaria, Euterpe edulis, Cinamomum glaziovii, Hyeronima alchornioides e Nectandra membranacea, sendo a maioria delas espécies mais tardias, indicadoras de estágios mais avançados de sucessão.

Peixoto et al. (2004), estudando uma mata antropizada em Pedra de Guaratiba (RJ), encontrou Pseudopiptadenia contorta como a segunda espécie mais importante, devido, igualmente, à sua alta dominância.

Astronium aculeatissimum se destacou entre as dez primeiras espécies em VI. Constata-se na área de estudo uma elevada densidade de indivíduos jovens dessa espécie, os quais foram excluídos da amostragem em função do critério de inclusão. Essa alta densidade é um indicativo de que essa espécie se regenera com facilidade em áreas alteradas. Esse fato pode ser comprovado em outros estudos realizados no estado, em matas alteradas antropicamente (BORÉM; OLIVEIRA-FILHO, 2002; NEVES, 1999; GUEDES, 1988), onde Astronium aculeatissimum aparece entre as primeiras espécies quanto ao valor de importância. Com isso, pode-se inferir que essa espécie seja indicadora de ambientes que estão em processo de regeneração após alteração antrópica em áreas sobre o domínio da Floresta Ombrófila Densa.

O remanescente estudado apresentou um dossel superior médio de 18 metros. Pseudopiptadenia contorta, Apuleia leiocarpa e Miconia cinnamomifolia apresentaram alguns indivíduos emergentes ultrapassando os 25 metros de altura.

O remanescente estudado apresentou a predominância de espécies secundárias iniciais (Figura 4), estando estas representadas pela maioria dos indivíduos amostrados (152 indivíduos). As espécies pioneiras foram as menos representativas, com apenas cinco espécies amostradas.

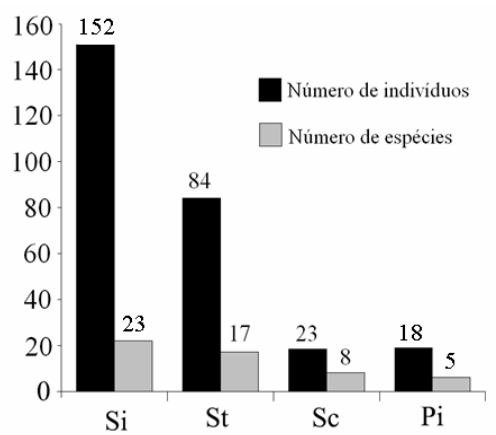

Figura 4. Distribuição das espécies e dos indivíduos amostrados na RPPN CEC Tinguá, bairro do Tinguá, Nova Iguaçu $(\mathrm{RJ})$, em categorias sucessionais. $\mathrm{Pi}=$ pioneira: $\mathrm{Si}=$ secundária inicial; $\mathrm{St}=$ secundária tardia; $\mathrm{Sc}=$ sem classificação.

Figure 4. Distribution of individuals and species sampled in RPPN CEC Tingua, district of Tingua, Nova Iguaçu $(\mathrm{RJ})$, in the succecional categories. $\mathrm{Pi}=$ pioneer species; $\mathrm{Si}=$ early secondary species; $\mathrm{St}=$ late secondary species; $\mathrm{Sc}=$ without data. 
Apesar da carência de estudos que indiquem o estado de conservação dos remanescentes florestais em áreas adjacentes à Reserva Biológica do Tinguá na Baixada Fluminense, provavelmente os recentes processos de fragmentação e distúrbios antrópicos na região acarretaram uma alteração florística nesses remanescentes. Um indicativo disso pode ser percebido ao se analisar a classificação sucessional das espécies inventariadas no CEC Tinguá, onde se observa o predomínio de espécies caracteristicamente secundárias iniciais, resultado este que, segundo Hubbel et al. (1999), é indicativo de matas perturbadas. No entanto, diante do resultado obtido (Figura 4), podemos perceber que o remanescente, apesar de ter sofrido intenso processo de perturbação pretérita, encontra-se, atualmente, em processo avançado de regeneração, caracterizado pela transição de espécies secundárias iniciais para secundárias tardias, o que vem a ser um indicativo de que a floresta estudada encontra-se em um estádio sucessional secundário.

\section{CONCLUSÕES}

A curva do coletor indicou que a amostragem foi bem representativa da diversidade local de espécies na área restrita à face norte do fragmento.

Myrtaceae, Fabaceae, Lauraceae e Euphorbiaceae apresentaram as maiores riquezas específicas. Esse padrão tem se repetido em estudos florísticos e/ou fitossociológicos realizados em diferentes regiões do estado do Rio de Janeiro.

O baixo índice de diversidade encontrado pode ser explicado, em parte, pelo histórico de alteração antrópica que o remanescente sofreu em um passado recente.

Percebe-se uma boa capacidade de recrutamento de indivíduos jovens na comunidade, fato esse indicado pela curva da distribuição diamétrica.

A comunidade estudada encontra-se em um processo de regeneração secundária avançado, caracterizado por uma grande quantidade de espécies secundárias iniciais, tais como Lacistema pubescens, Pseudopiptadenia contorta, Apuleia leiocarpa, Siparuna guianensis, Guapira opposita, Machaerium brasilense e Jacaranda puberula, entre outras.

Estudos dessa natureza são de grande importância, pois a análise dos dados de florística e fitossociologia combinados com grupos sucessionais das espécies locais formam uma base teórica de fundamental importância para subsidiar quaisquer iniciativas voltadas para a proteção, restauração e recuperação da vegetação local, uma vez que muitos modelos de recuperação e restauração ambiental se baseiam nesses parâmetros.

\section{AGRADECIMENTOS}

À Petrobrás, pelo financiamento do Programa Mata Atlântica (PMA), do qual esse estudo faz parte. Ao sr. Cristiano Camermam, proprietário da RPPN. Ao sr. Walter Silva, pelo auxílio em campo. Aos taxonomistas Alexandre Quinet, Ângela S. F. Vaz, Claudine M. Myssen, Elsie F. Guimarães, Haroldo C. de Lima, José Fernando A. Baumgratz e Marcelo C. Souza, pela identificação/confirmação de parte do material.

\section{REFERÊNCIAS}

APG II.. An update of APG classification for the orders and families of flowering plants. Botanical Journal of the Linnean Society, London, v. 141, p. 399-436, 2003.

BORÉM, R. T.; OLIVEIRA FILHO, A. T. Fitossociologia do estrato arbóreo em uma topossequência alterada de Mata Atlântica, no município de Silva Jardim-RJ, Brasil. Revista Árvore, Viçosa, MG, v. 26, n. 6, p. 727-742, 2002.

BRAZ, D. M.; MOURA, M. V. L. P.; ROSA, M. M. T. Chave de identificação para as espécies de Dicotiledôneas arbóreas da Reserva Biológica do Tinguá, RJ, com base em caracteres vegetativos. Acta Botanica Brasilica, Porto Alegre, v. 18, n. 2, p. 225-240, 2004. 
CARVALHO, F. A.; NASCIMENTO, M. T.; BRAGA, J. M. A. Estrutura e composição florística do estrato arbóreo de um remanescente de Mata Atântica submontana no município de Rio Bonito, RJ, Brasil (Mata Rio Vermelho). Revista Árvore, Viçosa, MG, v. 31, n. 4, p. 717-730, 2007.

CARVALHO, F. A.; NASCIMENTO, M. T.; ALVARENGA, J. M. Composição e riqueza florística do componente arbóreo da Floresta Atlântica submontana na região de Imbaú, Município de Silva Jardim, RJ. Acta Botanica. Brasilica, Porto Alegre, v. 20, n. 3, p. 727-740, 2006

CHRISTO, A. L.; GUEDES-BRUNI, R.; PINTO-SOBRINHO, F. A.; SILVA, A. G.; PEIXOTO, A. L. Structure of the shrub-arboreal componentes of an Atlântic Forest fragment on a hillock in the central lowland of Rio de Janeiro, Brazil. Interciencia, Catanduva, v. 34, n. 4, p. 232 a 239, 2009.

CUSTÓDIO, I. A. História da paisagem da região de Tinguá e arredores da Baía de Guanabara. Rio de Janeiro, RJ. 107 p. Dissertação (Mestrado em Botânica) - Jardim Botânico do Rio de Janeiro/ Escola Nacional de Botânica Tropical, 2007.

CORREDORES DE BIODIVERSIDADE DA MATA ATLÂNTICA. Corredores de biodiversidade da Serra do Mar. Disponível em: <http://www.corredores.org.br〉. Acesso em: 12/09/2006.

COSTA, L. G. S.; MANTOVANI, W. Dinâmica sucessional da floresta mesófila semidecídua em Piracicaba (SP). Oecologia Brasiliensis, Rio de Janeiro, v. 1, p. 291-305, 1995.

FUNDAÇÃO S. O. S. MATA ATLÂNTICA. Atlas dos Remanescentes Florestais e Ecossistemas Associados do Domínio da Mata Atlântica no período 1985 - 1990. São Paulo: Fundação SOS Mata Atlântica/INPE, 1993.

FUNDAÇÃO S. O. S. MATA ATLÂNTICA. Atlas da evolução dos remanescentes florestais e ecossistemas associados no domínio da Mata Atlântica no período 1995-2000. São Paulo: Fundação SOS Mata Atlântica/INPE/ISA, 2002.

GANDOLFI, S.; LEITÃO FILHO, H. F.; BEZERRA, C. L. F. Levantamento florístico e caráter sucessional das espécies arbustivo-arbóreas de uma floresta semidecídua no município de Guarulhos, SP. Revista Brasileira de Biologia, Rio de Janeiro, v. 55, p. 753-767, 1995.

GOLFARI. L.; MOOSMAYER, H. Manual de reflorestamento do estado do Rio de Janeiro. Rio de Janeiro: Banco de Desenvolvimento do Estado do Rio de Janeiro, Secretaria de Planejamento e Coordenação Geral. 1980. 382 p.

GUEDES-BRUNI, R. R. Composição, estrutura e similaridade de dossel em seis unidades fisionômicas de Mata Atlântica no Rio de Janeiro. 231 f. Tese (Doutorado em Ecologia) Universidade de São Paulo, São Paulo, 1998.

GUEDES-BRUNI, R. R.; SILVA-NETO, S. J.; MORIM, M. P.; MANTOVANI, W. Composição florística e estrutura de dossel em trecho de Floresta Ombrófila Densa atlântica sobre morrote mamelonar na Reserva Biológica de Poço das Antas, Silva Jardim, RJ. Rodriguesia, Rio de Janeiro, v. 57, p. 429442, 2006.

GUEDES-BRUNI, R. R.; PESSOA, S. V. A.; KURTZ, B. C. Florística e estrutura do componente arbustivo-arbóreo de um trecho preservado de floresta Montana na Reserva Ecológica de Macaé de Cima. In: LIMA, H. C. de; GUEDES- BRUNI, R. R. (Eds.). Serra de Macaé de Cima: Diversidade florística e conservação em Mata Atlântica. Rio de Janeiro: Instituto de Pesquisas Jardim Botânico do Rio de Janeiro. 1997. p. 127-145.

GUEDES, R. R. Composição florística e estrutura de um trecho de mata perturbada de baixada no Município de Magé, Rio de Janeiro. Arquivos do Jardim Botânico do Rio de Janeiro, Rio de Janeiro, v. 29 , p. $155-200,1988$.

HARPER, J. L. Population biology of plants. London: Academic, 1990. 892 p. 
HUBBEL, S. P.; FOSTER, R. B.; O.BRIEN, S. T.; HARMS, K. E.; CONDIT, R.; WECHSLER, B.; WRIGHT, S. J.; LAO, S. L. Light gaps disturbance, recruitment limitations and tree diversity in a neotropical forest. Science, Washington, DC, v. 283, p. 554-557, 1999.

IBGE. Vocabulário básico de recursos naturais e meio ambiente. 2. ed. Rio de Janeiro: Instituto Brasileiro de Geografia e Estatística, 2004. 332 p.

KURTZ, B. C.; ARAÚJO, D. S. D. Composição florística e estrutura do componente arbóreo de um trecho de Mata Atlântica na Estação Ecológica Estadual do Paraíso, Cachoeira de Macacú, Rio de Janeiro, Brasil. Rodriguesia, Rio de Janeiro, v. 51, n. 71/115, p. 69-112, 2000.

LIMA, H. C.; PESSOA, S. V.; GUEDES-BRUNI, R. R.; Moraes, L. F. D. de; GRANZOTTO, S. V.; IWAMOTO, S.; CIERO, J. Caracterização Fisionômico-Florística e Mapeamento da Vegetação da Reserva Biológica de Poço Das Antas, mun. Silva Jardim, RJ, Brasil. Rodriguesia, Rio de Janeiro, v. 57, p. 369-390, 2006.

LIMA, H. C. (Coord.). Paisagem e flora da Reserva Biológica do Tinguá: subsídios ao monitoramento da vegetação. Relatório Técnico. JBRJ. 2002. 25 p.

LOSS, A. C. C.; SILVA, A. G. Comportamento de forrageio de aves nectarívoras de Santa Teresa - ES. Natureza on line, Santa Teresa, ES, v. 3, p. 48-52, 2005.

MAGURRAN, A. E. Ecological diversity and its measurement. New Jersey: Princeton University Press. 1988.192 p.

MISSOURI BOTANICAL GARDEN. W3 Tropicos: Vascular Tropicos Nomenclatural Database. Disponível em: <http://www.mobot.org/W3T/Search/vast.html>. Acesso em: 13/11/2008.

MUELLER-DOMBOIS, D.; ELLEMBERG, H. Aims and methods of vegetation ecology. New York: J. Wiley \& Sons. 1974. 547 p.

NEVES, G. M. S. Florística e estrutura da comunidade arbustivo-arbórea em dois remanescentes de Floresta Atlântica secundária - Reserva Biológica de Poço das Antas, Silva Jardim, RJ. 115 f. Tese (Doutorado em Ecologia), Universidade Federal do Rio de Janeiro, Rio de Janeiro,1999.

OLIVEIRA-FILHO, A. T. TreeAtlan: Flora arbórea da Mata Atlântica domínios adjacentes: um banco de dados envolvendo geografia, diversidade e conservação. Disponível em: <http://www.treeatlan.dcf.ufla.br>. Acesso em: 18/02/2009.

OLIVEIRA, R. R. Ação antrópica e resultantes sobre a estrutura e composição da Mata Atlântica na Ilha Grande, RJ. Rodriguesia, Rio de Janeiro, v. 53, n. 82, p. 33-58, 2002.

OLIVEIRA, R. R.; ZAÚ, A. S.; LIMA, D. F.; SILVA, M. B. R.; VIANNA, M. C.; SODRÉ, D. O.; SAMPAIO, P. D. Significado ecológico da orientação de encostas no maciço da Tijuca, Rio de Janeiro. In: ESTEVES, F. A. (ed.) Oecologia brasiliensis: estrutura, funcionamento e manejo de ecossistemas brasileiros. Rio de Janeiro: Ed. UFRJ, 1995. v. 1, p. 523-541.

PEIXOTO, G. L., MARTINS, S. V.; SILVA, A. F.; SILVA, E. Composição florística do componente arbóreo de um trecho de Floresta Atlântica na Área de Proteção Ambiental da Serra da Capoeira Grande, Rio de Janeiro, RJ, Brasil. Acta Botanica Brasilica, Porto Alegre, v. 18, n. 1, p. 151-160, 2004.

PEIXOTO, G. L.; MARTINS, S. V.; SILVA, A, F.; SILVA, E. Estrutura do componente arbóreo de um trecho de Floresta Atlântica na Área de Proteção Ambiental da Serra da Capoeira Grande, Rio de Janeiro, RJ, Brasil. Acta Botanica Brasilica, Porto Alegre, v. 19, n. 3, p. 407-423, 2005.

PESSOA, S. V. A.; OLIVEIRA, R. R. Análise estrutural da vegetação arbórea em três fragmentos florestais na Reserva Biológica de Poço das Antas, Rio de Janeiro, Brasil. Rodriguesia, Rio de Janeiro, v. 57, n. 3, p. 391-411, 2006.

PESSOA, S. de V. A.; GUEDES-BRUNI, R. R.; KURTZ, B. C. Composição florística e estrutura do componente arbustivo-arbóreo de um trecho secundário de Floresta Montana na Reserva Ecológica de Macaé de Cima. In: LIMA, H. C.; GUEDES- BRUNI, R. R. (Eds.). Serra de Macaé de Cima: diversidade florística e conservação em Mata Atlântica. Rio de Janeiro: Instituto de Pesquisas Jardim Botânico do Rio de Janeiro, 1997. p. 147-167. 
PIELOU, E. C. Ecological diversity. New York: John Wiley \& Sons, 1975. 165 p.

PROGRAMA MATA ATLÂNTICA. Relatório Técnico. Jardim Botânico do Rio de Janeiro/IBAMA, Linhas de Ação em Botânica/CNPq, John D. \& Catherine T. MacArthur Foundation, Shell do Brasil S.A., Instituto Pró- Natura, 1992. 75 p.

RODRIGUES, H. C. Composição florística e fitossociológica de um trecho de Mata Atlântica na Reserva Biológica do Tinguá, Nova Iguaçu, Rio de Janeiro. 77 p. Dissertação ( Mestrado em Botânica) - Museu Nacional,Universidade Federal do Rio de janeiro, Rio de Janeiro, 1996.

SANTANA, C. A. A. Estrutura e dinâmica de florestas secundárias de encosta no município do Rio de Janeiro. 133 f. Dissertação ( Mestrado em ciências Florestais) - Universidade Federal Rural do Rio de Janeiro, Rio de Janeiro, 2000.

SHEPHERD, G. J. FITOPAC I. Manual do usuário. Campinas: Universidade Estadual de Campinas. Departamento de Botânica, 1996. 96 p.

SOBRINHO, F. A. P. Estudo etnobotânico de mateiros residentes no entorno de Unidades de Conservação no estado do Rio de Janeiro. 73 f. Dissertação (Mestrado em Botânica) - Instituto de Pesquisas Jardim Botânico do Rio de Janeiro, Escola Nacional de Botânica Tropical, Rio de Janeiro, 2007.

SCHILLING, A. C.; BATISTA, J. L. F. Curva de acumulação de espécies e suficiência amostral em florestas tropicais. Revista Brasileira de Botânica, São Paulo, v. 31, n. 1, p. 179-187, 2008.

SOLÓRZANO, A.; GUEDES-BRUNI, R. R.; OLIVEIRA, R. R. Composição florística e estrutura de dois trechos de Floresta Ombrófila Densa Submontana, no Parque Estadual da Pedra Branca, RJ. Revista Brasileira de Biociências, Porto Alegre, v. 5, p. 609-611, 2007.

WHITTAKER, R. H. Communities and ecosystems. New York: MacMillan, 1975. 387 p.

ZAR, J. H. Biostatistical analysis. New Jersey: Prentice Hall, 1999. 663 p. 
